\title{
EN TORNO AL ARISTOTELISMO DE GALILEO EN EL DIÁLOGO
}

\author{
Justiniano CASAS GONZÁLEZ \\ Departamento de Física de la Materia Condensada \\ Universidad de Zaragoza
}

\section{Introducción}

El objeto de este escrito es exponer un comentario crítico en torno a la Introducción que Antonio Beltrán Marí realiza a la edición del «Diálogo sobre los dos máximos sistemas del mundo ptolemaico y copernicanom en Alianza Editorial ${ }^{1}$. Me llamó la atención que la que puede considerarse como la edición "estandar" en castellano de una obra de tan alta relevancia, pudiera contener en algunos de los pasajes mas significativos de la Introducción lo que considero que son notables inexactitudes e interpretaciones del texto que no creo que se desprendan de una lectura sin prejuicios de la obra en cuestión.

Las consideraciones críticas que voy a presentar se dirigen grosso modo a dos aspectos distintos:

1) Interpretaciones que efectúa Beltrán del texto de Galileo argumentando que en buena medida está permeado por las ideas fundamentales aristotélicas. Se argumenta que la teoría aristotélica de los elementos está presente de manera primerísima en el pensamiento galileano. Según Beltrán, la teoría física del Diálogo supone que para Galileo el aire y el agua se comportan dinámicamente de manera diferente que la tierra, al modo al que lo hacen los distintos elementos en la física aristotélica. Este aristotelismo se extiende también a la física del movimiento de los graves. Argumentaré que esta interpretación del

1 Galilei. 
texto galileano es sumamente discutible. Ello no implica que en la obra de Galileo no pervivan elementos del pensamiento antiguo. Esto es manifiestamente así, como tendremos ocasión de comentar mas adelante. El Diálogo, con toda su grandeza, está plagado de argumentos que hoy día consideramos equivocados. Pero la significación que para Beltrán tienen algunos de ellos considero que está fuera de lugar. Comparto plenamente con el autor de la Introducción el que haya huido de hacer una presentación del texto de Galileo a la manera habitual en la que en muchas ocasiones se presenta a un público amplio las obras de los grandes científicos, narraciones en las que los héroes de la ciencia aparecen tocados con el arma de la infalibilidad ${ }^{2}$. Pero eso no debe llevar a que contradicciones o errores, significativos pero no obstante circunstanciales, acaben convirtiéndose en el polo interpretativo de un texto.

2) Por otra parte, Beltrán comete algunos errores al presentar elementos de lo que hoy día se considera conocimiento científico aceptado justamente para enjuiciar las inconsistencias del pensamiento de Galileo, llegándose a criticar el mismo apoyándose en consideraciones físicas inaceptables.

\section{La cuestión del aristotelismo en Copérnico y Galileo}

La argumentación de Beltrán resulta en bastantes ocasiones difícil de seguir. Comienza advirtiendo que «los argumentos que da Galileo con respecto al aire son los mismos que da Copérnico" (p. XLIX). Este último comparte con la tradición «la idea de un mundo organizado en esferas». En apoyo de esto se cita a Copérnico cuando responde a los tradicionales argumentos contra la rotación de la Tierra, proponiendo que las cosas que flotan en el aire siguen el movimento de la Tierra, «sea porque el aire próximo a la Tierra mezclado con materia acuosa o térrea, sigue la misma naturaleza que la Tierra, o sea porque el movimiento del aire es adquirido, que participa en la misma perpetua revolución y sin resistencia a causa de la contigüidad de la Tierra».

¿Pero que se quiere indicar cuando se habla de «un mundo elemental organizado en esferas»?. Si con ello se pretende decir que hay una esfera de tierra y sobre ella una capa de agua y sobre éstas dos una capa de aire, (éstas serían las esferas para Copérnico y Galileo) entonces nada hay que objetar a dicha descripción en

2 Véase por ejemplo GAMOw. 
el sentido de que es la forma habitual en la que cualquier tratado moderno de geología describiría grosso modo la estructura de la Tierra (una litosfera y una hidrosfera con tierra y agua y sobre éstas una atmósfera con sus distintas capas: troposfera, estratosfera, ionosfera). Esta es una descripción esencialmente topológica de la disposición de la materia de la Tierra y no tiene porque hacernos retrotraer a las esferas aristotélicas. Lo distintivo de las esferas aristotélicas es que

a) ellas constituyen el esquema fundamental en el que se explica el movimiento de los cuerpos y

b) se supone que la susbstancia sublunar está compuesta por los cuatro elementos correspondientes a las cuatro esferas.

Si, como parece obvio, no se puede atribuir específicamente a Aristóteles la creencia de que haya distintas capas de materia con características diferentes, la "idea del mundo sublunar organizado en esferas" por fuerza ha de referirse a que se comparte con la física aristotélica aspectos de (a) ó (b). Con respecto al apartado (b) algunos argumentos se ofrecen por parte de Beltrán según los cuales no está muy claro que Galileo considerara seriamente que la Luna podría estar compuesta por elementos distintos de los de la Tierra. No voy a entrar en este aspecto pues aunque creo que su punto de vista es cuando menos discutible, el mismo no constituye el nucleo de su argumentación. Por fuerza Beltrán debe de referirse a aspectos relativos al apartado (a).

Desde este punto de vista se puede entender como aristotélico en lo referente a dicho apartado el argumento que ofrece Copérnico mencionado mas arriba, pues dice que el aire próximo a la Tierra, mezclado con materia acuosa o térrea "sigue la misma naturaleza que la Tierra». Esto es plausible, pero hay que darse cuenta que ya Copérnico no propone esta solución como la única para resolver el problema, sino que sugiere a continuación que también los mismos fenómenos podrían explicarse porque la Tierra al rotar podría arrastrar el aire próximo a ella en su movimiento. Esta última causa nada tendría que ver con las propensiones naturales del aire etc., y se encontraría en otras coordenadas conceptuales. Coordenadas, que si uno se empeña, podría seguir llamando aristotélicas: el movimiento precisa de una fuerza aplicada constantemente para que tenga lugar. Cabría decir que Copérnico vacila entre caracterizar el movimiento del aire próximo a la superficie sólida de la Tierra como natural o como violento y seguir pensando que en cualquier caso se trata de aristotelismo, pero no creo que la cosa sea así de sencilla; si una explicación mira al pasado, la otra, con todas sus limitaciones, se aleja del mismo. El pensamiento moderno va a 
enjuiciar de de maneras bien distintas la dinámica del movimiento natural y la del violento. En la primera se explican los hechos acudiendo a lo que se caracterizó posteriormente como "cualidades ocultas" y fue rechazada por Descartes. El ideal cartesiano de explicación exige que ésta tenga lugar en "términos mecánicos", es decir, cuerpos que interactúan a través de choques, lo que da lugar a desplazamientos, arrastres, etc. Este ideal de explicación mecánica fue mantenido por la ciencia moderna a lo largo de los siglos XVIII y XIX donde se aplicó a las teorías de la electricidad, el calor y la luz ${ }^{3}$ y solo con la teoría cuántica acabará deshaciéndose, no sin reticencias, del mismo. El ideal de explicación mecánica entronca de esta manera con la teoría aristotélica del movimiento violento, pero el argumento copernicano del arrastre mira mas a la nueva física, con todas sus limitaciones, que a Aristóteles.

Volviendo al Galileo del Diálogo , es fácil constatar que éste maneja argumentaciones de carácter opuesto al tratar el problema del aire. El movimiento del mismo se explicará en general como debido al arrastre mecánico que ejerce la superficie irregular de la Tierra (lo que por otra parte se opone a su principio de inercia circular), si bien en ocasiones esta explicación aparece acompañada de otra fundada en la mezcla con vapores o exhalaciones terrestres que siguen de forma "natural» el movimiento diurno. Pero no creo que esta apelación que considero circunstancial a este tipo de argumentos deba de ser extendida a la teoría galileana del movimiento de los graves, donde son las nuevas ideas acerca del movimiento las que llevan la voz cantante. Beltrán lo intenta y el resultado no me parece convincente. Ello le lleva a sumir su argumentación en un conjunto de contradicciones que hacen que su lectura sea bastante dificultosa.

\section{El problema del movimiento del aire}

Comienza Beltrán su argumentación (p. XLIX) presentando el siguiente pasaje del Diálogo :

Añádase que es necesario que, al menos la parte del aire que está más baja que los montes más altos, sea arrastrada y hecha girar por la aspereza de la superficie terrestre, o bien que, en cuanto mezcla de muchos vapores y exhalaciones terrestres, observe naturalmente el movimiento diurno; lo cual no

3 Véase Harman. 
sucede con el aire que está en torno a la nave impulsada por los remos. Por lo cual el argumento de la nave no tiene fuerza de inferencia para la torre ${ }^{4}$.

Y a continuación:

La revolución diurna se da por movimiento propio y natural al globo terrestre, y en consecuencia a todas las partes y, en tanto impreso por la naturaleza, les es indeleble. Por ello, la piedra que está en la cima de la torre tiene como su instinto primario el girar en torno al centro de su todo en veinticuatro horas y ejerce ese talento natural eternamente, cualquiera que sea el estado en que sea puesta ${ }^{5}$.

El primer párrafo es, tal como Beltrán hace notar, muy similar al de Copérnico citado anteriormente y aparece la doble explicación para dar cuenta del movimiento del aire siguiendo a la Tierra. La cuestión vuelve a parecer en la cuarta jornada, donde se discute el problema de las mareas. Allí Galileo parece manejar sobre todo la explicación de carácter mecánico:

Por ello respecto al aire que circunda el globo terrestre, diría que, por su adherencia es arrastrado por el giro no menos que el agua, máxime la parte que está contenida en los recipientes que son las llanuras circundadas por los montes. Y podemos afirmar mucho mas razonablemente que esa porción es arrastrada en la rotación, arrebatada por la aspereza de la Tierra ${ }^{6}$.

Y más adelante:

Pero que un cuerpo de superficie áspera y montañosa, al girar sobre sí mismo, lleve consigo el aire contiguo al que van empujando sus prominencias es no ya verosímil, sino necesario... ${ }^{7}$

Aunque también:

Acabamos de decir, y repito lo añadido, que parecía que el aire, siendo un cuerpo tenue, fluido y no unido a la Tierra firmemente, no hubiera de tener la necesidad de obedecer el movimiento mas que en el caso en que la aspereza de la superficie terrestre lo arrebata y se lo lleva consigo una parte

4 Diálogo, p. 168. He seguido el texto por la propia traducción de Beltrán. Las referencias a las páginas corresponden a las de la edición en italiano a cargo de Favaro (Opere VII) que Beltrán mantienen en los márgenes, siguiendo así su propio criterio.

5 Ibid., p. 168.

6 Ibid., p. 464.

7 Ibid., p. 469. 
contigua a ella, que no sobrepasa en mucho las montañas mas altas. Dicha porción de aire habrá de ser tanto menos reticente a la rotación terrestre, cuanto que está llena de vapores humos y exhalaciones, materias todas que participan de las propiedades terrenas y, en consecuencia, adaptadas por su naturaleza a los mismos movimientos ${ }^{8}$.

Vemos por tanto que aunque se utiliza la explicación en términos de propidedades naturales, la misma aparece en general como subsidiaria de la mecánica. Pero la interpretación que ofrece Beltrán de estos textos es muy distinta. Par él todo debe de ser enjuiciado desde la teoría de los elementos, teniendo el "elemento aire" propiedades diferentes que el "elemento tierra». Es cierto que Galileo no pretende en ningún momento contestar a la pregunta de porque gira la Tierra sobre sí misma y en su movimiento anual. Este hecho es un punto de partida de sus argumentaciones, pero no creo que considerara que esos movimientos fueran algo específico de la Tierra en cuanto compuesta de "elemento tierra". En concreto, mas adelante pone en boca de Sagredo:

El [un opositor a Copérnico] me pregunta cuáles son los principios por los cuales el globo terrestre se mueve con movimiento anual en el zodíaco y con el diurno sobre el propio ecuador. Le respondo que éstos son algo similar a aquellos por los que Saturno se mueve a través del zodíaco en 30 años y sobre sí mismo en un tiempo mucho más breve en el plano de su ecuador, como nos lo muestra la aparición y ocultación de sus globos colaterales ${ }^{9}$.

Se puede interpretar este pasaje como que no hay para Galileo nada específico de la Tierra que la haga moverse como lo hace porque pudiera estar compuesta de "elemento tierra", pues lo que anima sus movimientos es similar a lo que anima los de Júpiter y es de suponer que los de los demas planetas. Pero si Beltrán tiene dudas acerca de la composición que para Galileo tiene la Luna, con mayor motivo debería extenderse la misma a los planetas. Cuando Beltrán interpreta a Galileo diciendo que "el elemento "aire" no tiene por naturaleza un movimiento circular que solo tiene el elemento "tierra». Más aun, el elemento aire tiene propiedades dinámicas distintas que el elemento Tierra (p.L) está forzando las cosas enormemente. En apoyo de las distintas propiedades dinámicas del aire como elemento se cita el siguiente párrafo:

8 Ibid., p. 465.

9 Ibid., p. 287. 
Puesto que como ya he dicho en otra ocasión, los cuerpos ligeros son mucho mas fáciles de ser movidos que los mas graves pero, al cesar la causa que los mueve, son tanto menos aptos para conservar el movimiento que se les ha impreso. Por lo cual el aire, siendo en sí mismo tenuísimo y ligerísimo es facilísimamente movible por la más mínima fuerza, pero también es ineptísimo para conservar el movimiento cuando también cesa el motor ${ }^{10}$

Pero esta propiedad del aire no parecer ser exclusiva del mismo en cuanto «elemento aire", sino de los cuerpos ligeros en general en oposición a los mas graves. Da la impresión que Galileo tiene en mente más bien el comportamiento habitual de los cuerpos en el seno de un fluido viscoso, tal como acontece con los cuerpos cuando cotidianamente se les comunica impulsos en la atmósfera (la pluma se para antes que la piedra cuando la lanzamos, etc.) y esta percepción la extiende al arrastre de toda la atmósfera por la parte sólida de la Tierra, olvidándose de su propio principio de inercia circular. ¿ No es mas razonable esta interpretación que el intento de llevarla a la teoría de los elementos.? Veremos más adelante que éste parece ser el curso del pensamiento de Galileo cuando se plantea el comportamiento del agua.

\section{El movimiento de los graves}

El segundo párrafo que cita Beltrán (nota $\mathrm{n}^{\circ}{ }^{5}$ ) parece indicar que la ideas acerca de las propiedades naturales son utilizadas por Galileo para explicar la caída de los graves. Sin embargo Beltrán parece no darse cuenta de cual es el contexto en el que aparece este pasaje en el Diálogo. En efecto, el texto traído a colación corresponde a una discusión en la cual Salviati pretende convencer a Simplicio de que una física aristotélica no tiene porqué estar en contra necesariamente de la idea de una Tierra en movimiento. Ello es evidente de toda la discusión. Comienza Salviati planteando claramente el problema:

Aristóteles dice que el argumento mas cierto contra la movilidad de la Tierra, es el que nosotros vemos que los proyectiles lanzados hacia arriba vuelven perpendicularmente por la misma línea al mismo lugar desde donde fueron lanzados y eso sucede aún cuando el movimiento llegue altísimo. Si la Tierra se moviese, esto no podría suceder, porque en el 
tiempo en que el proyectil se mueve hacia arriba y hacia abajo, separado de la Tierra, el lugar en el que se inició el movimiento se desplazaría, debido al giro de la Tierra, una distancia considerable hacia el levante y al caer el proyectil percutiría en Tierra a esa misma distancia lejos de dicho lugar ${ }^{11}$.

Este es el argumento que intenta atacar Galileo, utilizando como resulta evidente de la lectura de la discusión que viene a continuación, la propia física aristotélica, solo que cambiando las premisas de que al igual que el movimiento natural recto en una Tierra en reposo, explicaría la observación de la caída de la piedra rozando la torre, una Tierra en movimiento en la que el "movimiento natural" de los objetos fuese girar acompañando a la Tierra, explicaría análogamente la observación. $\mathrm{O}$ dicho en palabras del propio Salviati (nótese la utilización del subjuntivo):

Del mismo modo que, por haber considerado hasta ahora que el propiedad del globo terrestre permanecer inmóvil en torno a su centro, no he tenido ninguna dificultad u objeción en aceptar que cualquier pequeña parte suya también permanezca naturalmente en reposo, así también es preciso que si el instinto natural del globo terrestre fuese girar en veinticuatro horas, la inclinación intrínseca y natural de cada una de sus partes sea no el estar quieta sino seguir el mismo curso ${ }^{12}$.

La interpretación que se hace de esta parte del Diálogo sacando las afirmaciones de Salviati del contexto en las que son proferidas, le lleva al comentarista a asumir que la cosmología aristotélica, aunque aplicada al sistema de una Tierra en movimiento, está presente de forma primordial en el pensamiento de Galileo.

Una vez entendidos los supuestos teóricos de los que se parte, pasemos a analizar la discusión que se realiza en el Diálogo del vuelo de los pájaros, pieza central de la argumentación de Beltrán en favor de sus tesis. Al comentar los argumentos que utiliza Galileo, se dice que en los mismos se presenta una ambigüedad que va a tratar de sacar a la luz. Comienza indicando que al asunto se le ha prestado poca atención por parte de los comentaristas del Diálogo. Tan solo Clavelin, que al parecer no encuentra especial problema, le dedica un par de párrafos "en un lenguaje moderno» (sic):

11 Ibid., p. 164.

12 Ibid., p. 168 
"En realidad la dificultad se desvanece cuando se piensa en el comportamiento del aire, cuerpo grave el mismo, ligado mecánicamente a la tierra, posee el mismo «impeto" circular uniforme que todos los cuerpos materiales, y por esta razón acompaña a la tierra en su rotación. Así pues, cuando el pájaro o la nube que, por su parte, poseen el mismo impeto dejan de apoyarse en el suelo para apoyarse en el aire, no habrán de experimentar el menor cambio, ni sufrir la menor desviación bajo la influencia de un eventual movimiento diurno ${ }^{13}$.

Beltrán apostilla a esta cita: "Esta es, si acaso, nuestra explicación, pero desde luego no es la de Galileo». (p. LII. Cursivas en el original).

El texto de Clavelin es confuso por demás. Desde luego que no utiliza lo que podríamos llamar un "lenguaje moderno" al manejar conceptos como «impeto circular uniforme», que no se sabe muy bien que es. Beltrán parece interpretarlo como oponiéndolo a la doctrina del movimiento natural que según él utiliza Galileo: «Por lo que hemos visto hasta ahora, la constitución o naturaleza térrea de un cuerpo -es el caso de un pájaro o una nube- incluye como característica esencial de éste el que rote con la Tierra cada veinticuatro horas y no necesita recibir ningún impeto para hacerlo. El de rotación diurna es un movimiento natural. Es lo que nos ha dicho Galileo [...] (p. LII. Cursivas en el original). Es decir, que el movimiento de los pájaros según esta interpretación es un movimiento que precisa de una fuerza externa (impeto) para tener lugar, pues se opone así a la idea de movimiento natural. Pero si esa es nuestra explicación, según dice Beltrán, es que entendemos que los pájaros y nubes no se retrasan porque son empujados cuando están en el aire y ese empuje ha de venir forzosamente del propio aire. Esta parece ser en algunos momentos la explicación de Beltrán, aunque las cosas, por desgracia, nunca acaban de aclararse del todo como tendremos ocasión de ir comprobando.

Aparte de la explicación debida a la naturaleza térrea que, según el comentarista, maneja Galileo, se encuentra en el Diálogo una nueva explicación del movimiento de los pájaros distinta de la anterior que Beltrán pone de manifiesto:

Pero lo cierto es que el movimiento propio de los pájaros, quiero decir de su volar, no tiene nada que ver con el movimiento universal, al que no aporta ni ayuda ni estorbo. Lo que mantiene inalterado dicho movimiento

13 Cita de Beltrán a "La Philoslphie Naturelle de Galilée» París, 1968, cursivas mías. 
en los pájaros, es el mismo aire por el cual vagan que, siguiendo naturalmente la rotación de la Tierra, del mismo modo que lleva consigo las nubes, así también lleva consigo los pájaros y cualquier otra cosa que esté en él. Así pues, respecto a seguir a la tierra, los pájaros no tienen de que preocuparse, y por lo que a esto respecta podrían estar siempre dormidos ${ }^{14}$.

Está claro que en este pasaje Galileo atribuye el hecho de que los pájaros sigan a la Tierra a que son empujados por el aire, aunque posteriormente cambia el razonamiento y considera que la explicación debe ser similar a la ya utilizada para explicar el movimiento de los graves (Por cierto, aquí Galileo parece decir que el aire sigue naturalmente la rotación de la Tierra. Desaparecerían sus propiedades dinámicas específicas desde el punto de vista de los elementos).

Produce sin embargo confusión el comentario que se hace a dicho texto al indicar que a continuación Salviati «da una explicación que nos parece acorde con la nueva física que Galileo está introduciendo" pues "el movimiento diurno permanece siempre en los pájaros y los equipara en cuanto a graves a una piedra que dejamos caer desde una torre». (p. LIII). ¿Pero no se nos ha dicho hace poco que la explicación de que la piedra caiga pegada a la torre se fundamenta en la naturaleza térrea de la piedra? ¿Es ésta la nueva física que está introduciendo Galileo? Parece ser que sí, porque un poco mas adelante se vuelve a decir: "Por una parte se atribuye la rotación diurna de las nubes y pájaros a su naturaleza térrea. Por otra parte se atribuye dicha rotación al aire en el que están o vuelan". Da la impesión de que ahora se considera que el argumento erróneo es el del empuje del aire cuando hace poco uno parecía sacar en conclusión que esa era la explicación que se consideraba correcta, al menos era "nuestra explicación».

Las ambigüedades de Beltrán no acaban aquí, pues prosiguen cuando se pasa a comentar el famoso párrafo de Galileo de la descripción de un sistema inercial:

La causa de la total correspondencia de estos efectos es que el movimiento de la nave es común a todas las cosas contenidas en ésta incluyendo el aire, porque por eso dije yo que se, estuviese bajo cubierta.

Puesto que si se estuviese sobre ésta, al aire libre que no sigue el curso de la nave, se verian diferencias mas o menos notables en algunos de los efectos mencionados. No hay duda de que el humo quedaría atrás, como el aire mismo. Igualmente

14 Galilei, p. 193. 
las moscas y las mariposas, obstaculizadas por el aire, no podrían seguir el movimiento de la nave si se separasen de ésta por un espacio considerable. Pero si se mantuvieran próximas, puesto que la nave, por estar construida con anfractuosidades lleva consigo la parte próxima del aire, seguirían la nave sin dificultad o fatiga. Quizás por esta misma razón vemos que, cuando las caballerías corren, las moscas importunas y los tábanos siguen a los caballos volando ahora en ésta ahora en aquella parte de su cuerpo. Pero por lo que hace a las gotas que caen, la diferencia sería poquísima, y en los saltos y en los proyectiles graves totalmente imperceptibles ${ }^{15}$.

Y se apostilla: «Está claro que aquí, de nuevo, se insiste en el aire como responsable de que el humo y los animaluchos aéreos compartan el movimiento común de la nave y de los demás objetos». (p. LIV) Francamente, no se en que se fundamenta esta interpretación. Lo único que dice Galileo es que si la experiencia se realizara sobre la cubierta entonces el humo como el aire quedarían atrás y moscas y mariposas obstaculizadas no podrían seguir el movimiento de la nave, porque, efectivamente, en ese caso aparecería un viento relativo a la nave, que a buen seguro produciría los efectos indicados. Que Galileo no piensa que sea el aire el que empuja a los cuerpos bajo cubierta queda bien claro en el párrafo final en el que se dice que si se estuviera sobre cubierta los efectos sobre las gotas serían pequeñísimos y sobre los proyectiles prácticamente imperceptibles; a no ser que Beltrán considere que Galileo entiende que el principio del movimiento de moscas y mariposas es radicalmente distinto que el de gotas y piedras, cosa que no se aprecia en ningún momento.

Continúan los problemas en la discusión subsiguiente donde se trata de justificar que hay un paralelismo entre los casos Tierra-torre-piedra y navemástil-piedra por un lado y Tierra-aire pájaros y nave/camarote-aire-insectos por otro.

En el primer caso Beltrán se empeña en argumentar que Galileo considera que no son equivalentes: "En mi opinión está claro que si los casos Tierratorre-piedra y nave-mástil-piedra no son equivalentes....(p. LV) cuando justamente mediante una "trampa" dialéctica que Salviati tiende a Simplicio lo que de verdad argumenta es que sí que lo son. En efecto, no hay mas que echar un vistazo al texto para darse cuenta de que Salviati hace decir a Simplicio que ambas situaciones deben de ser equivalentes pese a las dificultades iniciales que

15 Ibíd., p. 213. Cursivas de Beltrán. 
le propone el propio Salviati (que son justamente en las que se basa Beltrán para su interpretación) para una vez logrado ese punto de partida argumentar la equivalencia:

SALV: [...] Y para no seguir teniéndoos, como suele decirse, sobre ascuas, decid Sr. Simplicio: ¿ estáis del todo convencido de que la experiencia de la nave cuadra tan bien a vuestro propósito que deba creerse razonablemente que lo que se ve sucede en ella debe suceder también en el globo terrestre?

SIMP: Hasta aquí me ha parecido que sí. $Y$ aunque vos habéis planteado algunas pequeñas diferencias, no me parecen tan importantes como para hacerme cambiar de parecer.

SALV.: Más bien deseo que continuéis en él y que tengáis por seguro que el efecto de la Tierra deba responder al de la nave, a condición de que si eso se mostrase perjudicial para vuestras necesidades, no se os ocurra cambiar de idea. [...] Y puesto que lo que ocurre en el caso de la nave debe igualmente suceder en el caso de la Tierra, del hecho de que la piedra caiga al pie de la torre se infiere necesariamente la inmovilidad del globo terrestre. ¿No es este vuestro razonamiento?

\section{$[\ldots]$}

SALV.: [...]. Porque cualquiera que la haga [la experiencia de la nave] hallará que la experiencia muestra todo lo contrario de lo que se ha escrito. Esto es, mostrará que la piedra cae siempre en el mismo lugar de la nave, tanto si está quieta como si se mueve con cualquier velocidad ${ }^{16}$.

El texto es lo suficientemente concluyente que no precisa de mas comentarios aclaratorios. Simplemente añadir que las dificultades que propone Salviati son razonables y que el viento relativo a la nave en movimiento podría producir tal vez un pequeño retraso de la piedra al ser soltada desde el mástil, pero Galileo está convencido de que, salvo por el inevitable viento en el caso de la nave, ambas experiencias son equivalentes.

Esto echa por tierra, naturalmente, la teoría de Beltrán de que Galileo atribuya a la "naturaleza térrea» de la piedra el que ésta caiga al pié de la torre. El porqué Galileo cree que la piedra en la experiencia de la nave caerá al pie del

$16 \quad$ Ibid., p. 168. 
mástil está muy claro de la discusión que realiza de que es lo que sucede cuando un jinete suelta una bola durante la carrera unas páginas antes del texto que acabamos de comentar:

"Mientras estáis sobre el caballo, ¿̨no corre vuestra mano y en consecuencia la bola tan veloz como el caballo mismo? Ciertamente sí, por tanto, con solo abrir la mano, la bola parte con el movimiento ya creado no por el movimiento particular de vuestro brazo, sino por el movimiento que depende del propio caballo, que os ha comunicado a vos, al brazo, a la mano, y finalmente a la bola ${ }^{17}$.

Añadase a ésto la teoría de la composición de movimientos que Galileo utiliza cuando se discuten los disparos de artillería y tendremos una idea muy clara de porque pensaba que no se retrasaría la piedra en el barco y porque no se retrasa en la torre. Es justamente al terminar esta discusión cuando le dice Sagredo a Simplicio:

....y si el Sr. Simplicio queda satisfecho con la solución del primer argumento contra la movilidad de la Tierra, respecto a los cuerpos que caen perpendicularmente, podríamos pasar a los otros ${ }^{18}$.

El análisis de las supuestas equivalencias e inequivalencias concluye con un párrafo en el que Beltrán vuelve a suscitar el asunto de las "naturalezas térreas» y que es enigmático por demás: « $\mathrm{El}$ único punto de equivalencia entre el caso de la Tierra-aire-pájaros y de la nave/camarote-aire-insectos consiste en que en ambos casos el aire está encerrado, en el camarote o entre las montañas y es empujado. Es obvio, pues que Galileo da importancia a este punto, en la medida en que le hace olvidar que mientras en el caso de la nave el aire del camarote comunica continuamente su movimiento a los insectos voladores y al humo, porque de lo contrario no se moverian siguiendo a la nave, en el caso de la Tierra los pájaros no necesitan dicho empuje porque se mueven por naturaleza siguiendo la rotación terrestre por su naturaleza térrea. Los insectos de la nave necesitan el aire para seguir la nave, los pájaros de la Tierra necesitan el aire sólo para volar, pero no para rotar con la Tierra. Los pájaros son Tierra, los insectos no son nave". (p. LV. Cursivas en el original). Aquí se encuentra resumido en una nuez toda la interpretación de Beltrán, pero el enigma al que

17 Ibid., p. 182.
18 Ibid., p. 188. 
me refiero se encuentra en el párrafo puesto en cursivas por el propio autor. A estas alturas ya no se sabe si entender que es Galileo el que piensa que efectivamente eso sería así, pero ha sufrido un olvido momentáneo, o es el propio Beltrán el que en realidad lo piensa. Uno no se queda muy tranquilo cuando el autor expone en el apartado siguiente y en el análisis de la teoría de las mareas de Galileo, su manejo de los principios de la física moderna.

Así, en el apartado siguiente que lleva por título «¿lujo y reflujo conceptual?» se dice lo siguiente cuando se comenta concepto del "Principio de inercia circular» galileano: «Se denomina así, porque por una parte hace la afirmación esencial del principio de inercia newtoniano, es decir la eterna continuidad del movimiento con velocidad uniforme, si ningún obstáculo exterior lo impide, aunque aquí se afirme sólo del circular". (p. LVIII, cursivas mías). El principio de inercia newtoniano, cuyo significado y alcance ha sido y sigue siendo objeto de análisis fue formulado por Newton en sus Principia de la forma siguiente: "Cada cuerpo permanece en su estado de reposo, o de movimiento rectilíneo uniforme, a menos que actúen fuerzas sobre él que le obliguen a cambiar este estado". Polémicas aparte acerca de las implicaciones de esta definición, lo que sí parece quedar claro es que un movimiento inercial debe de ser un movimiento rectilíneo. Todo movimiento no rectilíneo es un movimiento acelerado, aunque el módulo del vector velocidad no varie, pues necesariamente cambia la dirección del vector velocidad, y en consecuencia está sometido a una fuerza neta dirigida hacia el centro de curvatura de la trayectoria (la fuerza centrípeta). Aquí se confunde movimiento inercial, que se considera que es un movimiento en ausencia de fuerzas con un movimiento en el que se conseva la energía, que no tiene porque ser inercial, sino que las fuerzas que actúan sobre la partícula no realicen trabajo. Resulta por tanto equivocado afirmar (al menos en 1994) que el movimieno circular uniforme es un movimiento inercial, es decir, libre de fuerzas y que es un caso particular del principio general de inercia newtoniano. La confusión entre movimiento inercial y movimiento conservado es una de las fuentes de los problemas que aparecen en el apartado que dedica el autor al análisis de la teoría de las mareas de Galileo y que pasamos a analizar a continuación.

El movimiento del agua y la teoría de las mareas

El planteamiento de Beltrán en este asunto es otra vez el mismo: Sigue pensando que pervive la idea aristotélica de que el "elemento agua" no participa 
del movimiento diurno porque éste "sólo es natural al elemento tierra" (p.LXIV). Pero eso no es lo que dice Galileo. Galileo da una razón «física» al respecto:

El elemento agua [...] dado que no está unido ni pegado al globo terrestre como lo están sus partes sólidas, al contrario debido a su fluidez queda parcialmente "sui iuris" y libre ${ }^{19}$

La argumentación no parece tener que ver con la teoría de los elementos en el mundo sublunar. Más bien, parece que Galileo tiene en mente la imagen del comportamiento de una cantidad de líquido que avanza sobre una superficie pulida, estando en reposo relativo a la misma. Cuando se produce un frenazo del soporte, el líquido prosigue su camino debido a la débil adherencia. Cuando está contenido en un recipiente como una botella o una olla y se frena su movimiento, se producen agitaciones en el interior por idéntica causa. De hecho Galileo, cuando intenta hacer plausible la teoría de las mareas maneja un simil de este tipo:

Así pues, imaginemos que una de esas barcas [ que cruzan llenas de agua la laguna de Venecia ] viene con una velocidad moderada por la laguna, llevando plácidamente el agua de la que está llena, pero que después, o por encallar o por otro obstáculo que se le oponga, sea notablemente enlentecida. No por ello el agua contenida perderá, a la par que la barca el ímpetu ya adquirido, sino que consevándolo en sí, fluirá hacia la proa donde subirá perceptiblemente, bajando por la parte de popa. [...]. Este efecto es indiscutible $y$ claro y puede experimentarse en cualquier momento ${ }^{20}$.

¿También aquí el agua se comporta de esa manera por ser «elemento agua» distinto que la barca? ¿Hace falta invocar para algo la teoría de los elementos?. Beltrán lo hace porque de esa manera se salvaría la teoría de las mareas de la crítica a la que fue sometida por parte de otros autores.

El problema de la teoría de las mareas para estos críticos es que está en flagrante oposición con la descripción de un sistema inercial en el que las experiencias llevadas a cabo en el mismo no permitirían detectar si el sistema se encuentra en reposo o se mueve con movimiento rectilíneo uniforme que el

19 Ibid., p. 442.

20 Ibid., p. 451. 
propio Galileo ha introducido en el Diálogo en la segunda jornada y que ya hemos tenido ocasión de comentar. Lo que no parecen entender estos comentaristas es que un sistema rotatorio no es un sistema inercial y de hecho puede existir evidencia de la rotación. (Los giros de los vientos alrededor de los centros de alta y baja presión en sentido horario y antihorario respectivamente en el Hemisferio Norte y al revés en el Sur, la precesión en el plano de oscilación de los péndulos, es decir la conocida experiencia del "Péndulo de Foucault» y muchas otras experiencias cotidianas son explicadas satisfactoriamente como efectos de la rotación diurna de la Tierra). Se produce de esta manera una confusión para los sistemas inerciales similar a la ya comentada para el movimiento inercial. Beltrán reproduce un fragmento de Los sonámbulos de Koestler en el que parece que se llega a afirmar que «tanto la rotación de la Tierra como su revolución son inerciales, es decir, se perpetúan por sí mismas $y$, en consecuencia producen el mismo movimiento en agua y tierra, y una combinación de los mismos continúa dando como resultado el mismo impulso.», a lo que Beltrán apostilla : «La crítica es clara. Quizá demasiado clara.». Todas estas confusiones de Koestler, a Beltrán le parecen de lo más demoledoras. Pero tanto el uno como el otro están equivocados. Para salvar a Galileo de críticos tan agudos no hace falta echar mano de la teoría de los elementos, sino manejar un poco de física. Si su teoría de las mareas fracasa es por muchos otros motivos, pero no por el que se aduce por parte de Koestler. El modelo de Galileo, tal como él lo concibe, sí podría dar lugar a mareas ${ }^{21}$. (Y si no se convence el lector, súbase con una palangana llena de agua sobre las rodillas a una atracción de feria tipo "los troncos" en la que un carrusel gira con velocidad angular uniforme sobre otra plataforma giratoria a su vez con velocidad angular uniforme (sería un equivalente del modelo galileano). Es conveniente hacer la experiencia en verano).

21 El sistema Tierra-Sol es más complejo que el carrusel, pues según la física moderna interviene la fuerza gravitatoria, algo que Galileo no considera en ningún momento. Según la teoría newtoniana la Tierra está "cayendo" gravitacionalmente en el campo gravitatorio del Sol de manera que en su centro de gravedad se cancelan exactamente la fuerza gravitatoria y la centrífuga. Las mareas se explican adecuadamente al ser ligeramente más débil la fuerza gravitacional que la centrífuga (al sumarse la velocidad de la órbita en torno al sol con la de rotación diurna) en los puntos de la Tierra más alejados del Sol y suceder lo contrario en los más próximos. (gravedad mayor y velocidades que se restan). Es innegable un «aire de familia» con la teoría galileana. La desmotración matemática de la existencia de mareas en el modelo de Galileo se desarrolla en el apéndice. 
También es inadecuada la forma en que Beltrán intenta enmendarle la plana a Galileo cuando entra a argumentar porqué su modelo es, aún echando mano de la teoría de los elementos, insatisfactorio.(lo que por otra parte no tiene vuelta de hoja). Se nos dice que la teoría de Galileo daría lugar a «maremotos y otros desastres geológicos» pues "al amanecer debería producirse un frenazo de 2000 millas/hora y al anochecer una aceleración equivalente». (p. LXV). En primer lugar, millas/hora no son unidades de aceleración sino de velocidad. La aceleración se define como la tasa de cambio de velocidad y sus dimensiones serán en las unidades elegidas por Beltrán de millas/(hora) ${ }^{2}$.En este sentido se pueden producir cambios en la velocidad de la magnitud indicada por Beltrán y la aceleración ser muy pequeña. En el caso que nos ocupa la aceleración sería del orden de:

$$
\mathrm{a}=\Delta \mathrm{v} / \Delta \mathrm{t} \approx 3000 \mathrm{Km} / \mathrm{hora} / 43.200 \text { segundos (mitad de un día) }
$$

que resulta ser del orden de $0.016 \mathrm{~m} / \mathrm{segundo}^{2}$, es decir aproximadamente 1000 veces mas pequeña que la aceleración de la gravedad en la superficie de la Tierra $\left(9.8 \mathrm{~m} / \mathrm{segundo}^{2}\right)$ lo que no debería de provocar maremotos. (Incidentalmente, esa aceleración es real, solo que dirigida hacia en centro de la Tierra (salvo por la pequeña aceleración asociada al movimiento anual, que introduciría una componente tangencial responsable de las mareas en el modelo de Galileo. Ver el cálculo de la aceleración tangencial en el apéndice).y ello explica el cambio en el valor de la gravedad aparente sobre la superficie de la Tierra con la latitud). Beltrán se empeña en suponer que el cambio de velocidad se produciría de manera casi brusca y a partir de ahí uno se puede imaginar cualquier cosa.

\section{Conclusiones}

A modo de resumen: He tratado de poner de manifiesto que la interpretación que Beltrán hace del pensamiento galileano en su estudio introductorio al Diálogo, se basa en un lectura del mismo en la que no se ha llegado a entender adecuadamente el cambio conceptual que representa esta obra con respecto a la física copernicana y aristotélica. Los elementos de apoyo para esta interpretación suponen una comprensión poco adecuada de la obra en su conjunto, sacando fuera de contexto algunos pasajes e interpretando equivocadamente otros. Ello no está en contradicción con el reconocimiento de que en ocasiones Galileo maneja concepciones del pensamiento antiguo y argumentaciones 
incorrectas, pero que en nada desvirtúan el carácter de ruptura conceptual que sin duda se plasma en esta obra.

Por otra parte, las argumentaciones utilizadas son a menudo oscuras y difíciles de seguir. En algunos casos no se utilizan adecuadamente los principios de la teoría física moderna.

\section{Agradecimientos}

El Profesor Carlos Solís del Departamento de Lógica y Filosofía de la Ciencia de la U.N.E.D. tuvo la amabilidad de leer una versión preliminar del presente escrito y me hizo llegar muchas sugerencia valiosas. Por suspuesto que las posibles incorrecciones que permanezcan en el mismo son de mi entera responsabilidad.

\section{Apéndice}

Para demostrar que en el modelo de Galileo se producirian mareas basta con probar que aparecería una aceleración tangencial a la superficie de la Tierra, lo que daría lugar a la correspondiente fuerza de inercia que desplazaría el agua. Consideramos el caso particular de un punto sobre el Ecuador terrestre.

Sean w y W las velocidades angulares de rotación de la Tierra en su movimiento diurno y anual respectivamente. Eligiendo adecuadamente el origen de coordenadas cartesianas para fijar la posición de un punto sobre el Ecuador de la Tierra, las componentes catesianas del vector posición para un sistema de referencia con origen en el centro del Sol pueden escribirse como:

$$
x=R \operatorname{sen}(\Omega t)+r \operatorname{sen}[(\Omega+\omega) t] y=R \cos (\Omega t)+r \cos [(\Omega+\omega) t]
$$

donde $\mathrm{R}$ y $\mathrm{r}$ representan las distancias del centro del Sol al centro de la Tierra y el radio de la Tierra respectivamente; $t$ representa el tiempo.

Derivando dos veces con respecto al tiempo se obtiene la expresión para las componentes de la aceleración a lo largo de las direcciones $\mathrm{x}$ e y: 


$$
\begin{aligned}
& \ddot{x}=-\Omega^{2} R \operatorname{sen}(\omega t)-(\Omega+\omega)^{2} \operatorname{rsen}[(\Omega+\omega) t] \\
& \ddot{y}=-\Omega^{2} R \cos (\omega t)-(\Omega+\omega)^{2} \operatorname{rcos}[(\Omega+\omega) t]
\end{aligned}
$$

La aceleración tangencial a la superficie de la Tierra se calcula proyectando:

$$
a_{t}=\ddot{x} \cos [(\Omega+\omega) t]-\ddot{y} \operatorname{sen}[(\Omega+\omega) t]
$$

Substituyendo los valores para las componentes cartesianas de la aceleración, (2) en (3) y operando se obtiene:

$$
a_{t}=\Omega^{2} \operatorname{Rsen}(\omega t)
$$

Nótese que si $\Omega=0$, es decir si solo existiera movimiento de rotación diurno, la aceleración tangencial se anularía. La única componente sería la normal dirigida al centro de la Tierra, lo que no daría lugar a mareas. Si $\Omega$ es del orden de $2 \times 10^{-7}$ rad.seg $^{-1}, \omega=7.2 \times 10^{-5} \mathrm{rad}^{-\mathrm{seg}^{-1}}$ y tomamos $\mathrm{R}=150 \times 10^{9}$ metros, se obtiene un valor máximo de esta aceleración tangencial de $6.3 \times 10$ ${ }^{3} \mathrm{~m} . \mathrm{seg}^{-2}$, unas 1500 veces mas pequeña que la aceleración de la gravedad en la superficie de la Tierra. Utilizando un valor para $\mathrm{R}$ de $4.6 \times 10^{6}$ millas, el valor manejado por Galileo, la aceleración tangencial máxima sería unas 20 veces mas pequeña, es decir, unas 30000 veces inferior a la de la gravedad, lo que no daría lugar, desde luego, a los mencionado maremotos. No obstante, la altura de las mareas que predice el modelo de Galileo sí que tal vez podría ser considerable en los grandes océanos, pues el cociente entre la altura de la marea y la longitud del mar debería ser del orden del cociente entre $a_{t}$ y la aceleración de la gravedad en la superficie de la Tierra; Para un océano como el Atlántico con una anchura de unos miles de kilómitros a la altura del Ecuador, las mareas deberían llegar a ser del orden de unos cientos de metros, (decenas de metros con las magnitudes de las que disponía Galileo) si bien el agua iría subiendo en el transcurso de 6 horas, lo que ciertamente no provocaría maremotos, aunque enormes extensiones de terreno se verían anegadas. (Por otra parte, el modelo predice entre otras cosas que por ejemplo en la península itálica, cuando fuera marea alta en la costa tirrena debería ser baja en la adriática y viceversa. No creo que haga falta insistir mas en las inadecuaciones de esta teoría). 


\section{Bibliografía}

HARMAN, P. M. (1990): Energía, fuerza y materia. El desarrollo conceptual de la fisica del siglo XIX. (Alianza, Madrid).

GaLILEI, Galileo (1994): Diálogo sobre los dos máximos sistemas del mundo ptolemaico y copernicano. (Alianza, Madrid). Traducción, introducción y notas a cargo de Antonio BELTRÁN MARI.

GAMOw, George (1980): Biografia de la fisica. (Alianza, Madrid). 